\title{
Single Nucleotide Polymorphism-Based Chromosomal Microarray Evaluation of Hydatidiform Moles: A US National Reference Laboratory Experience
}

\author{
Arturo Anguiano ${ }^{1,2^{*}}$, Boris T. Wang ${ }^{*}$, Kylin Y. Lammers ${ }^{2}$, Loretta W. Mahon ${ }^{2}$, Nicole Truitt ${ }^{3}$, \\ Lindsay Dohany ${ }^{3}$, Fatih Z. Boyar ${ }^{1 \#}$ \\ ${ }^{1}$ Cytogenetics Department, Quest Diagnostics Nichols Institute, San Juan Capistrano, CA, USA \\ ${ }^{2}$ Medical Affairs Department, Quest Diagnostics, San Juan Capistrano, CA, USA \\ ${ }^{3}$ Laboratory Medical Affairs, Progenity, Inc., Ann Arbor, MI, USA \\ Email: Arturo.L.Anguiano@questdiagnostics.com, Boris.T.Wang@questdiagnostics.com, \\ Kylin.Y.Lammers@questdiagnostics.com, Loretta.W.Mahon@questdiagnostics.com, Nicole.truitt@progenity.com, \\ Lindsay.Dohany@progenity.com, ${ }^{*}$ Fatih.Z.Boyar@questdiagnostics.com
}

How to cite this paper: Anguiano, A., Wang, B.T., Lammers, K.Y., Mahon, L.W., Truitt, N., Dohany, L. and Boyar, F.Z. (2020) Single Nucleotide Polymorphism-Based Chromosomal Microarray Evaluation of Hydatidiform Moles: A US National Reference Laboratory Experience. Open Journal of Obstetrics and Gynecology, 10, 1122-1134. https://doi.org/10.4236/ojog.2020.1080105

Received: July 21, 2020

Accepted: August 23, 2020

Published: August 26, 2020

Copyright ( 2020 by author(s) and Scientific Research Publishing Inc. This work is licensed under the Creative Commons Attribution International License (CC BY 4.0).

http://creativecommons.org/licenses/by/4.0/ (c) (i) Open Access

\begin{abstract}
Objectives: This retrospective study evaluated 1) benefits of single nucleotide polymorphism (SNP)-based chromosomal microarrays (CMAs) in the diagnosis of complete hydatidiform mole (CHM) and partial HM (PHM) in products of conception (POC) and amniotic fluid (AF) specimens, and 2) frequency of whole-genome uniparental disomy (wgUPD) and triploidy in POC and AF specimens received at a US national reference laboratory. Methods: We reviewed consecutive 2138 POC and 3230 AF specimens and identified the cases with wgUPD and triploidy which are associated with molar pregnancy. Results: Of 2138 consecutive POC specimens tested, SNP-based CMA detected wgUPD in $10(0.47 \%)$ and triploidy in 84 (3.93\%). Of the 10 wgUPD cases, 9 (90\%) were confirmed as CHM. Of 3230 consecutive AF specimens, the array detected wgUPD in 1 case $(0.03 \%)$ and triploidy in 11 (0.34\%). Conclusions: SNP-based microarray allows detection of wgUPD in POC and AF specimens at a US national reference laboratory. Correctly diagnosing $\mathrm{HM}$ and differentiating $\mathrm{CHM}$ from $\mathrm{PHM}$ are important for clinical management. The effective SNP-based CMA detection of wgUPD in CHM may enable physicians to monitor patients at risk for gestational trophoblastic disease and neoplasm. Conventional chromosome analysis of POC has a high failure rate, cannot be performed on formalin-fixed paraffin embedded samples, and cannot detect wgUPD. Further multi-institutional collaborative assessment
\end{abstract}

${ }^{*}$ Co-first authors with equal contribution. 
on accuracy, cost-effectiveness, and adequate access to SNP-based CMA, may lead this testing platform to be considered as the first-tier analysis tool for POC specimens, including those showing PHM or CHM.

\section{Keywords}

Complete Hydatidiform Mole (CHM), Gestational Trophoblastic Disease (GTD), Gestational Trophoblastic Neoplasm (GTN), Partial Hydatidiform Mole (PHM), Triploidy, Whole Genome Uniparental Disomy (wgUPD)

\section{Introduction}

Molar pregnancy, or hydatidiform mole (HM), is an abnormal pregnancy characterized by overgrowth of trophoblastic cells. HM can be classified into two types: complete hydatidiform mole (CHM) and partial hydatidiform mole (PHM). The incidence of CHM is approximately 1 per 1500 (0.07\%) pregnancies, while the incidence of PHM may be as high as 1 in $700(0.14 \%)$ pregnancies [1]. HMs can also recur as either CHM or PHM, with recurrence risk being approximately $1 \%$; if a second mole occurs, the recurrence risk increases to approximately $20 \%$ [1].

Both CHM and PHM can develop into persistent gestational trophoblastic disease (GTD) [2]. GTD may also transform into a gestational trophoblastic neoplasm (GTN). GTNs have an excellent prognostic outcome following chemotherapy, and early detection of GTD-associated genomic aberrations such as whole-genome UPD (wgUPD) may prove vital to alert clinicians to the risk of developing GTNs [1] [3] [4] [5]. The risks of developing an invasive mole or choriocarcinoma are higher for CHM (15\% and 3\%, respectively) than PHM (0.5\% and $0.1 \%$, respectively) [1] [2] [6]. Thus, correctly diagnosing HM and differentiating CHM from PHM are important for clinical management.

CHM is caused by a paternal wgUPD [1]. Paternal disomy is usually caused by an empty ovum being fertilized by 2 sperm (23, X and 23, Y, or 23, X and 23, X) or by a single $23, \mathrm{X}$ sperm that doubles. In contrast, $\mathrm{PHM}$ is most often caused by an ovum being fertilized by 2 sperm (androgenetic), yielding a triploid complement; triploidy with an underlying digyny (gynogenetic) can also occur but is caused by a diploid ovum being fertilized with a haploid sperm. PHM correlates more strongly with androgenetic triploidy than with gynogenetic triploidy [7]. Rare cases of HM with tetraploidy have been reported in the literature [8]. Most molar pregnancies with tetraploid cells appear to be produced by somatic endoreduplications, while a minority originates from a tetraploid zygote [8].

Diagnosis of HM is conventionally based on a history of lack of fetal movement, a pelvic examination, an ultrasound study (US), or a blood test to look for a high level of beta human chorionic gonadotropin (hCG) [3] [4] [9]. One disadvantage of the US is that pregnancies affected by some non-molar chromosomal abnormalities (such as paternal UPD for 11p) may demonstrate abnormal 
chorionic villous morphology that mimics an HM [10]. However, single nucleotide polymorphism (SNP)-based chromosomal microarray (CMA) can detect UPD for chromosome 11.

SNP-based CMA uses SNP data to determine the number and combination of alleles, as well as region-of-homozygosity $(\mathrm{ROH})$ status throughout the genome. Thus, it can accurately detect both wgUPD and triploidy, in addition to single-chromosome UPDs, chromosomal numerical abnormalities, submicroscopic copy number variations, and consanguinity. Furthermore, SNP-based-CMA has a very low failure rate, eliminates time required for cell culture, eliminates need for metaphase cells, has a shorter turnaround time than chromosome study, and can also be used with formalin-fixed paraffin embedded (FFPE) specimens from products of conception (POC) [11] [12] [13].

Other assays used to diagnose HM have some shortcomings. Chromosome analysis of cultured cells has been a routine practice to evaluate POC. This method can detect triploidy associated with PHM but cannot detect wgUPD associated with CHM; further, the chromosome analysis to evaluate POC has a test failure rate of approximately 25\% [11]. An SNP-based CMA platform can be used to diagnose HM, but a non-SNP-based CMA can incorrectly indicate a triploid genome as diploid because of normalization of the copy-number data. Furthermore, a CHM evaluated with a non-SNP-based CMA would be interpreted as a diploid genome with no insight as to the underlying wgUPD. Thus, if only conventional karyotyping methods and/or copy-number-based (non-SNP-based) CMA analyses are implemented, a CHM may be misdiagnosed as a non-molar pregnancy. The SNP-based CMA platform is the most informative tool to simultaneously evaluate dosage abnormalities (triploidy) associated with PHM, as well as the ROH seen in wgUPD that are associated with CHM [14].

This retrospective study evaluated 1) the benefits of SNP-based CMA in the diagnosis of CHM and PHM in POC and amniotic fluid specimens and 2) the frequency of wgUPD and triploidy in POC and amniotic fluid specimens received for testing with SNP-based CMA at a national reference laboratory.

\section{Materials and Methods}

This study included de-identified results from 2138 consecutive POC and 3230 consecutive AF specimens submitted to Quest Diagnostics to rule out genetic alterations.

All POC specimens (including placental and fetal tissues) were examined, dissected, cleaned and rinsed 3 times in culture medium to remove maternal deciduous tissue before set-up for culture and direct DNA extraction from chorionic villi or fetal parts. The amniotic fluid specimens submitted for prenatal diagnosis were processed in accordance with standard laboratory protocols.

Genomic DNA was extracted from primarily the uncultured POC and amniotic fluid specimens by using standard DNA extraction methods (QIAGEN QIAamp DNA blood mini or micro kit using QIAcube or QIAsymphony, Ger- 
mantown, MD USA). The CytoScan ${ }^{\mathrm{TM}}$ HD microarray contains over 2.67 million probes (1.9 million copy number probes and 750,000 SNP probes), with an average interprobe distance of 1150 base pairs (GeneChip ${ }^{\mathrm{TM}}$ probe Array, Affymetrix ${ }^{\circledR}$, Inc., Santa Clara, CA, USA; currently ThermoFisher Scientific, Life Technologies, Carlsbad, CA, USA). These specimens were then analyzed by oligo-SNP CMA (CytoScan ${ }^{\circledR}$ HD Array, Affymetrix ${ }^{\circledR}$, Inc., Santa Clara, CA, USA; currently ThermoFisher Scientific, Life Technologies, Carlsbad, CA USA). For genome-wide screening, thresholds were $>200 \mathrm{~kb}$ for gains, $>50 \mathrm{~kb}$ for losses, and $>10 \mathrm{Mb}$ for regions of homozygosity $(\mathrm{ROH})$. Analysis of the results was performed using the ChAS software.

Based on an empirical testing dataset, quality control metrics have been determined by Affymetrix, currently ThermoFisher Scientific Life Technologies, as follows: Median of the absolute values of all pairwise differences (MAPD) is a global measure of the variation of all microarray probes across the genome. It represents the median of the distribution of changes in log2 ratios between adjacent probes. Since it measures differences between adjacent probes, it is a measure of short-range noise in the microarray data. Array data with MAPD values greater than 0.25 is inadequate to provide reliable copy number calls. Waviness $\mathrm{SD}$ is a global measure of variation of microarray probes that is insensitive to short-range variation and focuses on long-range variation. Array data with waviness $\mathrm{SD}$ values greater than 0.12 has either sample or processing batch effects that will reduce the quality of the copy number calls. Elevated waviness SD is not always an indication of too much noise. Elevated waviness with acceptable MAPD and SNPQC metrics can occur in samples with many copy number changes or very large regions of change. It is therefore advised to check the data when observing elevated waviness with good MAPD and SNPQC values. SNPQC is a measure of how well genotype alleles are resolved in the microarray data. Array data with SNPQC values less than 15 is of less quality than is required to meet genotyping QC standards.

The CytoScan ${ }^{\circledR}$ HD Array testing platform was fully validated in our setting and externally reviewed and approved by the New York Department of Health (non-published, internal records). The validation included intra-assay and inter-assay performance assessment in abnormal result identification. In every instance, the abnormality was identified. Across both inter- and intra-assay tests, the MAPD values had a mean of 0.175 and a range of $0.151-0.201$ (threshold < $0.25)$, the Waviness SD values had a mean of 0.082 and a range of $0.078-0.102$ (threshold < 0.12) and SNPQC values had mean of 24.012 and a range of 19.249 27.016 (threshold > 15). Additional quality control measures include bi-annual monitoring of performance of scanners, with replicates of a sample with a known abnormality; in every instance the known abnormality has been confirmed.

All laboratory results and clinical information used in this study were anonymized before the authors had access to them. Thus, they were deemed exempt from the requirement for consent. 


\section{Results}

Among the 2138 POC specimens tested using SNP-based microarray analysis, wgUPD (examples in Figure 1 and Figure 2) was identified in $10(0.47 \%)$ and triploidy (example in Figure 3) was identified in 84 (3.93\%). Thus, the prevalence of triploidy was 8 times higher than that of wgUPD in POC specimens submitted for testing.

Of 10 cases of wgUPD detected in POC specimens, there was available clinical information confirming a CHM on 8 cases with whole-genome uniparental isodisomy (wgUPiD) and one case with whole-genome uniparental heterodisomy (wgUPhD). Only 4 out of the 10 cases with wgUPD had p57 [KIP2] staining information available and all four cases were reported with negative p57 [KIP2] staining result (Table 1). CMA identified wgUPiD in 9 of these 10 specimens and $\mathrm{wgUPhD}_{\text {in }}$ the other. The case identified as $\mathrm{wgUPhD}$ (patient number 9 in Table 1) was also associated with CHM, as evidenced by the appearance of hydropic villi and no detectable fetus in the surgical pathology report.

Of the 84 triploidy cases detected in POC specimens, clinical information was only available for 22 . Of these 22 cases, 12 (55\%) were associated with the histopathological diagnosis of PHM (Supplementary Table S1). Of the remaining 10 cases, 1 was an HM without further diagnosis and 9 were inconsistent with HM.

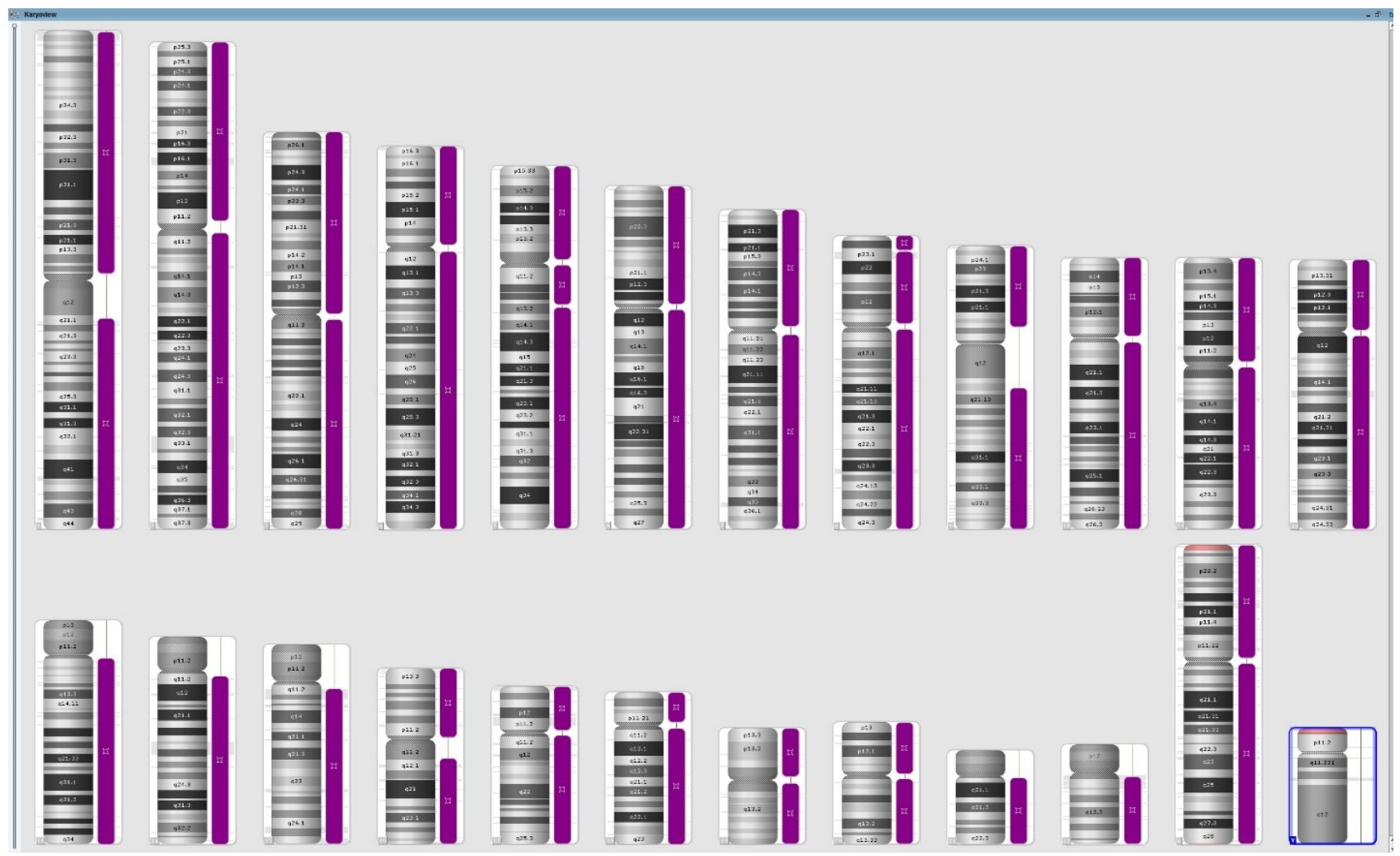

Figure 1. An example (patient number 1 in Table 1) of complete hydatidiform mole with a female diploid karyotype. By single-nucleotide polymorphism (SNP)-based microarray, the homozygosity bars (purple) next to every chromosome of the whole genome indicate the presence of whole-genome uniparental isodisomy. This represents a classical way a complete hydatidiform mole forms, in which doubling of a single 23, X sperm has occurred after fertilization with an "empty" ovum. 


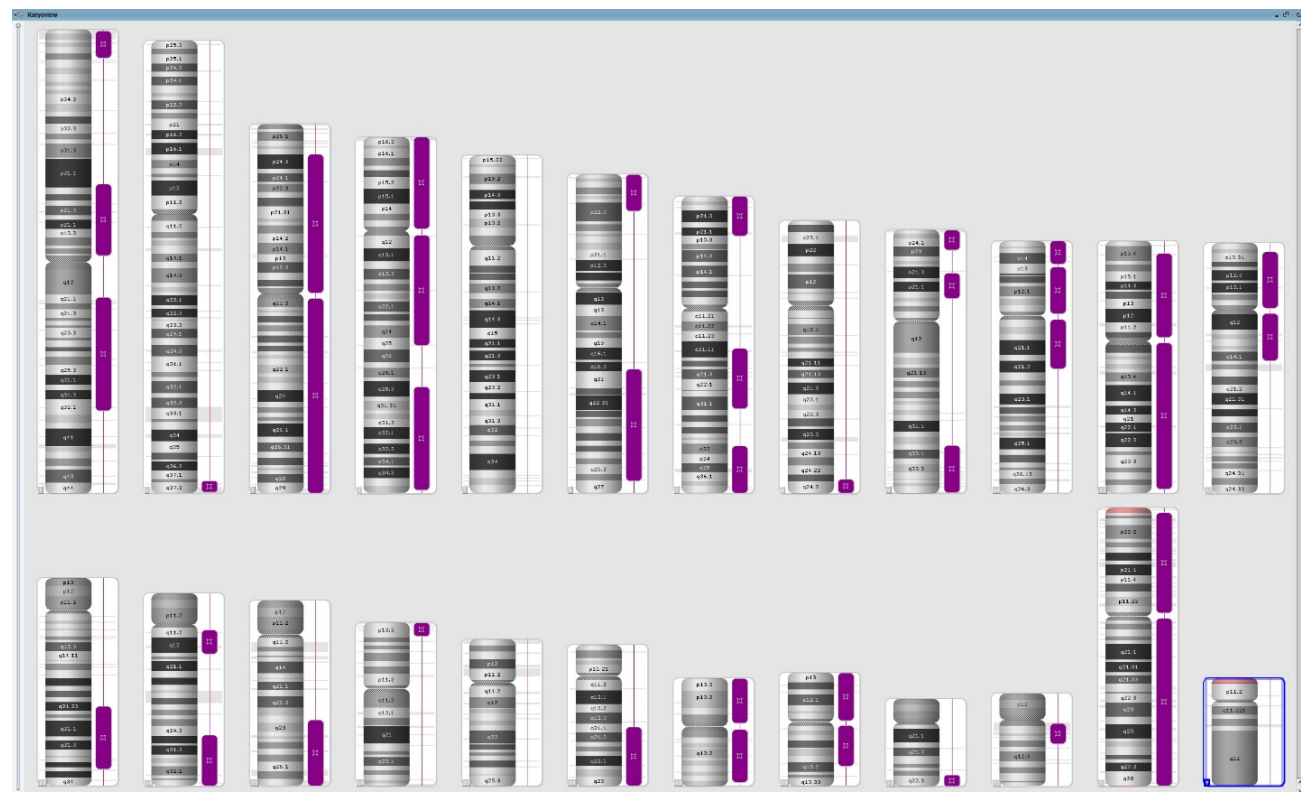

Figure 2. An example (patient number 9 in Table 1) of complete hydatidiform mole with a male diploid karyotype (as evidenced by three lines of the pseudo-autosomal region (PAR), not shown in this image). Multiple regions of homozygosity (purple bars in variable sizes totaling $1231 \mathrm{MB}$ ) next to most chromosomes were present in this SNP-based microarray data. This finding suggests that whole-genome uniparental heterodisomy (wgUPhD) resulted from a situation where multiple cross-over events between the paired chromosomes have occurred. This represents a rare type of fertilization in which an "empty" ovum was fertilized by two sperm (X and Y) [20].

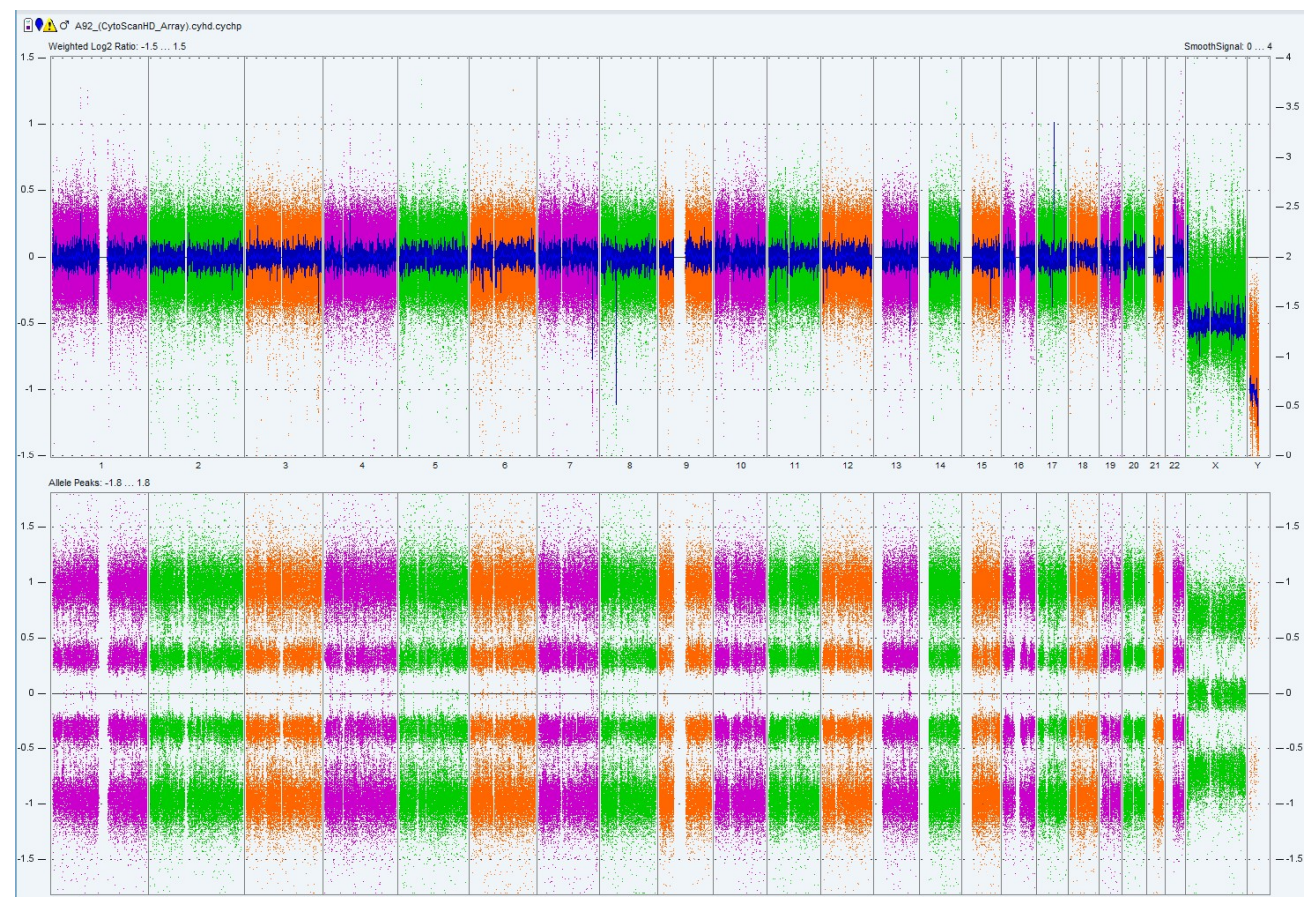

Figure 3. An example (patient number 18 in Supplementary Table S1) of partial hydatidiform mole with a triploid karyotype $(69, \mathrm{XXY})$ in a male product of conception specimen. Note that the copy-number state and the log2 ratio tracks (upper) of non-SNP based (copy number based) Affymetrix array show two copies for all autosomes, while the SNP tracks reflect the three allele combinations as four tracks ( $\mathrm{AAA}, \mathrm{AAB}, \mathrm{ABB}, \mathrm{BBB}$ ) (bottom) in triploidy. 
Table 1. Clinical information provided in the 10 cases positive for wgUPD (wgUPiD or wgUPhD) among 2138 consecutive product of conception (POC) specimens analyzed with SNP-based CMA.

\begin{tabular}{|c|c|c|c|c|c|}
\hline $\begin{array}{l}\text { Patient } \\
\text { Number }\end{array}$ & PATHOLOGY RESULT & hCG LEVEL (GA) & IHC p57(KIP2) RESULT & ISCN NOMENCLATURE & $\begin{array}{l}\text { UPD } \\
\text { TYPE }\end{array}$ \\
\hline 1 & Consistent with $\mathrm{CHM}$ & $\begin{array}{c}\text { Elevated } \\
\text { (GA not identified) }\end{array}$ & $\begin{array}{l}\text { Negative stain, consistent } \\
\text { with complete mole }\end{array}$ & $\operatorname{arr}(1-22, \mathrm{X}) \mathrm{x} 2 \mathrm{hmz}$ & wgUPiD \\
\hline 2 & Consistent with CHM & NA & NA & $\operatorname{arr}(1-22, \mathrm{X}) \mathrm{x} 2 \mathrm{hmz}$ & wgUPiD \\
\hline 3 & Consistent with $\mathrm{CHM}$ & Elevated (12 weeks) & NA & $\operatorname{arr}(1-22, \mathrm{X}) \mathrm{x} 2 \mathrm{hmz}$ & wgUPiD \\
\hline 4 & $\begin{array}{l}\text { No pathology study done, } \\
\text { no information on molar pregnancy }\end{array}$ & Normal & NA & $\operatorname{arr}(1-22, \mathrm{X}) \mathrm{x} 2 \mathrm{hmz}$ & wgUPiD \\
\hline 5 & Choriocarcinoma & $\begin{array}{c}\text { Elevated (levels not } \\
\text { provided; GA-9 weeks) }\end{array}$ & NA & $\operatorname{arr}(1-22, \mathrm{X}) \times 2 \mathrm{hmz}$ & wgUPiD \\
\hline 6 & Consistent with $\mathrm{CHM}$ & Normal & $\begin{array}{l}\text { Negative stain, consistent } \\
\text { with complete mole }\end{array}$ & $\operatorname{arr}(1-22, \mathrm{X}) \times 2 \mathrm{hmz}$ & wgUPiD \\
\hline 7 & Degenerate POC; consistent with $\mathrm{CHM}$ & NA & NA & $\operatorname{arr}(1-22, X) \times 2 h m z$ & wgUPiD \\
\hline 8 & Consistent with CHM & NA & $\begin{array}{l}\text { Negative stain, consistent } \\
\text { with complete mole }\end{array}$ & $\operatorname{arr}(1-22, X) \times 2 h m z$ & wgUPiD \\
\hline 9 & $\begin{array}{l}\text { Consistent with CHM, hydropic villi and } \\
\text { no detectable fetus }\end{array}$ & NA & $\begin{array}{l}\text { Negative stain, consistent } \\
\text { with complete mole }\end{array}$ & $\begin{array}{c}\operatorname{arr}(1-22) \times 2 \mathrm{hmz} / \mathrm{htz},(\mathrm{X}, \\
\mathrm{Y}) \mathrm{x} 1 \text { (total sum of ROHs > } \\
5 \mathrm{Mb} \text { is } 1,231 \mathrm{Mb})^{\dagger}\end{array}$ & wgUPhD \\
\hline 10 & $\begin{array}{l}\text { Consistent with CHM (cystic placenta, no } \\
\text { identifiable embryonic fetal tissue) }\end{array}$ & NA & NA & $\operatorname{arr}(1-22, X) \times 2 h m z$ & wgUPiD \\
\hline
\end{tabular}

GA: gestational age; wgUPiD: whole-genome uniparental isodisomy; wgUPhD: whole-genome uniparental heterodisomy; ROH: regions of homozygosity; NA: no information available. The hCG level was considered elevated when it was $>100,000 \mathrm{mIU} / \mathrm{mL}$. ${ }^{+}$The ISCN long form for patient number 9 in Table 1 : $\operatorname{arr}[\mathrm{hg} 19] 1 \mathrm{p} 31.1 \mathrm{q} 32.1(83,129,232-204,717,211) \times 2 \mathrm{hmz}, 1 \mathrm{p} 36.33 \mathrm{p} 36.21(882,802-15,195,444) \times 2 \mathrm{hmz}, 2 \mathrm{q} 37.2 \mathrm{q} 37.3(236,835,656-242,775,910) \times 2 \mathrm{hmz}$, 3p25.1q29(16, 285, 940-197, 851, 260)x2 hmz, 4p16.3q25(46, 690-111, 743, 853)x2 hmz, 4q28.3q35.2(134, 314, 405-189, 433, 290)x2 hmz, 6q16.3q26(104, 699, 789-164, 392, 986)x2 hmz, 6p25.3p22.3(184, 718-19, 705, 844)x2 hmz, 7p22.3p15.3(44, 166-21, 164, 766)x2 hmz, 7q33q36.3(134, 121, 268-159, 119, 220)x2 hmz, 7q21.11q31.1(81, 736, 266-113, 741, 105)x2 hmz, 8q24.23q24.3(139, 051, 845-146, 292, 734)x2 hmz, 9q32q34.3(115, 928, 565-141, 025, 328)x2 hmz, 9p24.3p23(192, 128-10, 523, 383)x2 hmz, 9p21.3p13.2(23, 426, 270-36, 885, 919)x2 hmz, 10p15.3p13(95, 661-12, 383, 617)x2 hmz, 10p13q21.3(14, 207, 914-68, 769, 550)x2 hmz, 11p15.4q25(6, 894, 738-132, 773, 772)x2 hmz, 12p13.32q14.1(4, 984, 600-62, 871, 827)x2 hmz, 13q21.33q33.2(71, 151, 636-105, 665, 669)x2 hmz, 14q11.2q13.1(20, 511, 672-33, 537, 514)x2 hmz, 14q24.3q32.33(79, 184, 349-107, 285, 437)x2 hmz, 15q22.31q26.3(66, 089, 282-102, 429, 049)x2 hmz, 16p13.3(89, 560-7, 115, 496)x2 hmz, 18q21.1q23(45, 667, 056-78, 014, 582)x2 hmz, 19p13.3q13.43(260, 911-57, 160, 596)x2 hmz, 20p13q13.2(61, 794-51, 804, 954)x2 hmz, 21q22.2q22.3(42, 143, 502-48, 084, 820)x2 hmz, 22q11.1q12.1(16, 877, 134-27, 819, 961)x2 hmz.

Among the 3230 amniotic fluid specimens, wgUPD was identified only in 1 $(0.03 \%)$ and triploidy was identified in $11(0.34 \%)$. Thus, the prevalence of triploidy was 11 times higher than that of wgUPD. Of the 11 triploidy cases, 6 had available clinical information and $1(17 \%)$ of these was reported to be a PHM (Supplementary Table S2).

\section{Discussion}

SNP-based CMA is $100 \%$ sensitive for detection of wgUPD and triploidy. This retrospective study of data from a national reference laboratory demonstrates that SNP-based CMA can be used for detection of wgUPD and triploidy in POC and AF specimens in a clinical laboratory setting. In this setting, the prevalence of triploidy was over 8 times higher than that of wgUPD in POC specimens ( $4 \%$ vs $\sim 0.5 \%)$ and over 11 times higher in AF specimens (0.34\% vs $0.03 \%)$. These 
results indicate that SNP-based CMA may allow physicians to detect wgUPD, and thus monitor patients at risk of GTD and GTN.

Compared to POC results of another group [12], the prevalence of wgUPD in our study was similar ( $0.47 \%$ vs. $0.5 \%)$, but the prevalence of triploidy in our study was lower (3.9\% vs. $6.3 \%)$. Aside from the rare recurrent $\mathrm{CHM}$ with a biparental diploid karyotype, which is an autosomal recessive disease called familial recurrent HM (FRHM) due to biallelic mutations in 2 genes: NLRP7 at $19 q 13.42$ [15] [16], and more rarely, KHDC3L at 6q13 [17], the genetic abnormalities associated with cases of triploidy with PHM, and the wgUPD associated with CHM can be reliably detected by oligo-SNP based CMA; correlation with clinical findings, histopathological features, p57 (KIP2) immunohistochemistry, and genotyping results may ensure accurate classification in equivocal cases [14].

One limitation of this study is that the approach may underestimate actual occurrence rates of $\mathrm{HM}$, because a substantial proportion of pregnancies involving wgUPD or triploidy are likely to abort spontaneously before testing in the first trimester. HMs, whether complete or partial, have an underlying genetic cause. Upon the clinical suspicion of CHM, a p57 (KIP2) expression study has been used to identify androgenetic cell lines in POC [4]. However, approximately $10 \%$ of CHM results are atypical for p57 (KIP2), which can lead to false-negative results [5]. The histological evaluation of PHM and CHM remains as a diagnostic challenge in certain settings and in various clinical situations [18] [19]. On the other hand, a false-positive result can occur if paternal UPD 11p is present, which results in loss of p57 (KIP2) expression [10]. Determination of the underlying etiology in such cases may be difficult without a molecular assay that can also detect UPD 11p, such as molecular genotyping with short tandem repeat (STR) loci, or an SNP-based CMA. Xie et al. reported some cases of CHM and PHM that were not suspected by experienced obstetricians [14]. Those unsuspected cases were diagnosed with an SNP-based CMA; the detection of such unsuspected cases may prove vital to alert clinicians of the risk of those patients to develop GTN.

\section{Conclusion}

SNP-based microarray allowed effective detection of wgUPD in POC and AF specimens at a US national reference laboratory. Correctly diagnosing HM and differentiating CHM from PHM are important for clinical management. The effective SNP-based CMA detection of wgUPD in CHM may enable physicians to monitor patients at risk for gestational trophoblastic disease and neoplasm. Conventional chromosome analysis of POC has a high failure rate, cannot be performed on formalin-fixed paraffin embedded samples, and cannot detect wgUPD. Our current findings, our previously reported experience with SNP-based arrays for analysis of POC at a national reference laboratory, and the experience of other groups [12] [14] [21], may further pave the way towards a multi-institutional collaborative assessment on accuracy, cost-effectiveness, and 
adequate access to SNP-based CMA, which may lead this testing platform to be considered as the first-tier analysis tool for POC specimens, including those showing PHM or CHM.

\section{Acknowledgements}

The authors thank Andrew B. Hellman, PhD, and Jeff Radcliff (Quest Diagnostics) for assistance in editorial review of the manuscript. Joey Kelly, Morteza Hemmat, Jack Wang and Renius Owen were involved in data collection.

\section{Funding Statement}

Not applicable.

\section{Authors' Contributions}

A.A. and B.T.W. were involved in the study concept and design, analysis and interpretation of data, and drafted the manuscript and revised it critically for important intellectual content. L.W.M., K.Y.L., N.T. and L.D. were involved in data collection and the analysis and interpretation of data. F.Z.B. was involved in the study concept and design and revised it critically for important intellectual content. All authors read and approved the final manuscript.

\section{Conflicts of Interest}

A.A, B.T.W, K.Y.L., L.W.M, and F.Z.B. are employees of Quest Diagnostics and have stock ownership. N.T. and L.D. are employees of Progenity.

\section{References}

[1] Gardner, R.J.M. and Amor, D.J. (2018) Gardner and Sutherland's Chromosome Abnormalities and Genetic Counseling. 5th Edition, Oxford University Press, Oxford. https://doi.org/10.1093/med/9780199329007.001.0001

[2] Seckl, M.J., Fisher, R.A., Salerno, G.A., Rees, H., Paradinas, F.J., Foskett, M. and Newlands, E.S. (2000) Choriocarcinoma and Partial Hydatidiform Moles. Lancet, 356, 36-39. https://doi.org/10.1016/S0140-6736(00)02432-6

[3] Seckl, M.J., Sebire, N.J., Fisher, R.A., Golfier, F., Massuger, L., Sessa, C. and ESMO Guidelines Working Group (2013) Gestational Trophoblastic Disease: ESMO Clinical Practice Guidelines for Diagnosis, Treatment and Follow-Up. Annals of Oncology, 24, 39-50. https://doi.org/10.1093/annonc/mdt345

[4] Banet, N., DeScipio, C., Murphy, K.M., Beierl, K., Adams, E., Vang, R. and Ronnett, B.M. (2014) Characteristics of Hydatidiform Moles: Analysis of a Prospective Series with p57 Immunohistochemistry and Molecular Genotyping. Modern Pathology, 27, 238-254. https://doi.org/10.1038/modpathol.2013.143

[5] Duffy, L., Zhang, L., Sheath, K., Love, D.R. and George, A.M. (2015) The Diagnosis of Choriocarcinoma in Molar Pregnancies: A Revised Approach in Clinical Testing. Journal of Clinical Medicine \& Research, 7, 961-966. https://doi.org/10.14740/jocmr2236w

[6] American Cancer Society. Gestational Trophoblastic Disease. https://www.cancer.org/cancer/gestational-trophoblastic-disease.html 
[7] Zaragoza, M.V., Surti, U., Redline, R.W., Millie, E., Chakravarti, A. and Hassold, T.J. (2000) Parental Origin and Phenotype of Triploidy in Spontaneous Abortions: Predominance of Diandry and Association with the Partial Hydatidiform Mole. American Journal of Human Genetics, 66, 1807-1820. https://doi.org/10.1086/302951

[8] Sundvall, L., Lund, H., Niemann, I., Jensen, U.B., Bolund, L. and Sunde, L. (2013) Tetraploidy in hydatidiform moles. Human Reproduction, 28, 2010-2020. https://doi.org/10.1093/humrep/det132

[9] Berkowitz, R.S. and Goldstein, D.P. (2009) Molar Pregnancy. The New England Journal of Medicine, 360, 1639-1645. https://doi.org/10.1056/NEJMcp0900696

[10] Sebire, N.J., May, P.C., Kaur, B., Seckl, M.J. and Fisher, R.A. (2016) Abnormal Villous Morphology Mimicking a Hydatidiform Mole Associated with Paternal Trisomy of Chromosomes 3, 7, 8 and Unipaternal Disomy of Chromosome 11. Diagnostic Pathology, 11, Article No. 20. https://doi.org/10.1186/s13000-016-0471-9

[11] Wang, B.T., Chong, T.P., Boyar, F.Z, Kopita, K.A., Ross, L.P., El-Naggar, M.M., Sahoo, T., Wang, J., Hemmat, M., Haddadin, M.H., Owen, R. and Anguiano, A.L. (2014) Abnormalities in Spontaneous Abortions Detected by G-Banding and Chromosomal Microarray Analysis (CMA) at a National Reference Laboratory. Molecular Cytogenetics, 7, Article No. 33. https://doi.org/10.1186/1755-8166-7-33

[12] Sahoo, T., Dzidic, N., Strecker, M.N., Commander, S., Travis, M.K., Doherty, C., Tyson, R.W., Mendoza, A.E., Stephenson, M., Dise, C.A., Benito, C.W., Ziadie, M.S. and Hovanes, K. (2017) Comprehensive Genetic Analysis of Pregnancy Loss by Chromosomal Microarrays: Outcomes, Benefits, and Challenges. Genetics in Medicine, 19, 83-89. https://doi.org/10.1038/gim.2016.69

[13] Gliem, T.J. and Aypar, U. (2017) Development of a Chromosomal Microarray Test for the Detection of Abnormalities in Formalin-Fixed, Paraffin-Embedded Products of Conception Specimens. Journal of Molecular Diagnostics, 19, 843-847. https://doi.org/10.1016/j.jmoldx.2017.07.001

[14] Xie, Y., Pei, X., Dong, Y., Wu, H., Wu, J., Shi, H., Zhuang, X., Sun, X. and He, J. (2016) Single Nucleotide Polymorphism-Based Microarray Analysis for the Diagnosis of Hydatidiform Moles. Molecular Medicine Reports, 14, 137-144. https://doi.org/10.3892/mmr.2016.5211

[15] Fisher, R.A., Hodges, M.D. and Newlands, E.S. (2004) Familial Recurrent Hydatidiform Mole: A Review. Journal of Reproductive Medicine, 49, 595-601.

[16] Murdoch, S., Djuric, U., Mazha, B., Seoud, M., Khan, R., Kuick, R., Bagga, R., Kircheisen, R., Ao, A., Ratti, B., Hanash, S., Rouleau, G.A. and Slim, R. (2006) Mutations in NALP7 Cause Recurrent Hydatidiform Moles and Reproductive Wastage in Humans. Nature Genetics, 38, 300-302. https://doi.org/10.1038/ng1740

[17] Kalogiannidis, I., Kalinderi, K., Kalinderis, M., Miliaras, D., Tarlatzis, B. and Athanasiadis, A. (2018) Recurrent Complete Hydatidiform Mole: Where We Are, Is There a Safe Gestational Horizon? Opinion and Mini-Review. Journal of Assisted Reproduction and Genetics, 35, 967-973.

https://doi.org/10.1007/s10815-018-1202-9

[18] Stevens, F.T., Katzorke, N., Tempfer, C. Kreimer, U., Bizjak, G.I., Fleisch, M.C. and Fehm, T.N. (2015) Gestational Trophoblastic Disorders: An Update in 2015. Geburtshilfe und Frauenheilkunde, 75, 1043-1050. https://doi.org/10.1055/s-0035-1558054

[19] Izadi-Mood, N., Sarmadi, S., Tayebivaljozi, R., Mohammadi-Zia, F. and Farhadi, M. (2015) Flow Cytometric DNA Analysis and Histopathologic Re-Evaluation of Paraf- 
fin Embedded Samples from Hydatidiform Moles and Hydropic Abortions. International Journal of Fertility \& Sterility, 9, 322-328.

[20] Usui, H., Nakabayashi, K., Maehara, K., Hata, K. and Shozu, M. (2019) Genome-Wide Single Nucleotide Polymorphism Array Analysis Unveils the Origin of Heterozygous Androgenetic Complete Moles. Scientific Reports, 9, Article No. 12542. https://doi.org/10.1038/s41598-019-49047-7

[21] Maisenbacher, M.K., Merrion, K. and Kutteh, W.H. (2019) Single-Nucleotide Polymorphism Microarray Detects Molar Pregnancies in 3\% of Miscarriages. Fertility and Sterility, 112, 700-706. https://doi.org/10.1016/j.fertnstert.2019.06.015 


\section{Supplementary}

Table S1. Clinical information provided in the 22 POC cases positive for triploidy.

\begin{tabular}{|c|c|c|c|}
\hline $\begin{array}{l}\text { Patient } \\
\text { Number }\end{array}$ & PATHOLOGY RESULTS & hCG LEVEL & ISCN NOMENCLATURE \\
\hline 11 & Chorionic villi present, consistent with PHM & Elevated & $\operatorname{arr}(1-22, \mathrm{X}) \mathrm{x} 3$ \\
\hline 12 & Consistent with PHM & Elevated & $\operatorname{arr}(1-22, X) \times 3$ \\
\hline 13 & Consistent with PHM & N/A & $\operatorname{arr}(1-22, X) \times 3$ \\
\hline 14 & Consistent with MP & N/A & $\operatorname{arr}(1-22) \times 3,(\mathrm{X}) \times 2,(\mathrm{Y}) \mathrm{x} 1$ \\
\hline 15 & $\begin{array}{l}\text { Consistent with PHM; patient had a chorio- } \\
\text { carcinoma and a hysterectomy in } 2014 \text {. }\end{array}$ & N/A & $\operatorname{arr}(1-22, \mathrm{X}) \mathrm{x} 3$ \\
\hline 16 & Consistent with PHM & Elevated & $\operatorname{arr}(1-22, X) x 3$ \\
\hline 17 & Consistent with PHM & N/A & $\operatorname{arr}(1-22, \mathrm{X}) \mathrm{x} 3$ \\
\hline 18 & Consistent with PHM & N/A & $\operatorname{arr}(1-22) \times 3,(X) \times 2,(Y) \times 1$ \\
\hline 19 & Consistent with PHM & $\mathrm{N} / \mathrm{A}$ & $\operatorname{arr}(1-22, X) \times 3$ \\
\hline 20 & Consistent with PHM & N/A & $\operatorname{arr}(1-22, X) \times 3$ \\
\hline 21 & $\begin{array}{l}\text { Immature chorionic villi present; } \\
\text { Consistent with PHM }\end{array}$ & N/A & $\operatorname{arr}(1-22, \mathrm{X}) \times 3$ \\
\hline 22 & $\begin{array}{l}\text { Immature chorionic villi present; } \\
\text { Consistent with PHM }\end{array}$ & N/A & $\operatorname{arr}(1-22, \mathrm{X}) \times 3$ \\
\hline 23 & $\begin{array}{l}\text { Immature chorionic villi present; } \\
\text { Consistent with PHM }\end{array}$ & N/A & $\operatorname{arr}(1-22) \times 3,(\mathrm{X}) \times 2,(\mathrm{Y}) \mathrm{x} 1$ \\
\hline 24 & Not conclusive with MP & N/A & $\operatorname{arr}(1-22) \times 3,(\mathrm{X}) \times 2,(\mathrm{Y}) \mathrm{x} 1$ \\
\hline 25 & Not conclusive with MP & N/A & $\operatorname{arr}(1-22) \times 3,(\mathrm{X}) \times 2,(\mathrm{Y}) \mathrm{x} 1$ \\
\hline 26 & Not conclusive with MP & N/A & $\operatorname{arr}(1-22) \times 3,(\mathrm{X}) \times 2,(\mathrm{Y}) \times 1$ \\
\hline 27 & Not conclusive with MP & N/A & $\operatorname{arr}(1-22, \mathrm{X}) \times 3$ \\
\hline 28 & Not conclusive with MP & N/A & $\operatorname{arr}(1-22, X) \times 3$ \\
\hline 29 & Not conclusive with MP & N/A & $\operatorname{arr}(1-22, \mathrm{X}) \mathrm{x} 3$ \\
\hline 30 & Not conclusive with MP & Normal & $\begin{array}{l}\operatorname{arr}(1-12,14-22) \times 3,(13) \times 4 \\
(\mathrm{X}) \times 2,(\mathrm{Y}) \times 1\end{array}$ \\
\hline 31 & Not conclusive with MP & Normal & $\operatorname{arr}(1-22, \mathrm{X}) \mathrm{x} 3$ \\
\hline 32 & Not conclusive with MP & Normal & $\operatorname{arr}(1-22, X) \times 3$ \\
\hline
\end{tabular}

Among the 22 POC cases positive for triploidy, 12/22 (55\%) were reported to be PHM, and one of the 12 cases had previously developed into choriocarcinoma. Please note that among the 6 POC cases provided with hCG levels, 3/6 (50\%) were reported to be hCG elevated.

Table S2. Clinical information provided in 6 amniotic fluid cases.

\begin{tabular}{|c|c|c|c|}
\hline Patient ID & PATHOLOGY RESULTS & hCG LEVEL & ISCN NOMENCLATURE \\
\hline 33 & Consistent with PHM & Elevated hCG & $\operatorname{arr}(1-22, X) \times 3$ \\
\hline 34 & Pathology of placenta normal & N/A & $\operatorname{arr}(1-22, X) x 3$ \\
\hline 35 & $\begin{array}{l}\text { Placenta unremarkable on ultrasound; } \\
\text { no further f/u information available }\end{array}$ & N/A & $\operatorname{arr}(1-22) \times 3,(\mathrm{X}) \mathrm{x} 2,(\mathrm{Y}) \mathrm{x} 1$ \\
\hline
\end{tabular}




\section{Continued}

\begin{tabular}{clcc}
\hline 36 & $\begin{array}{l}\text { Macerated fetal parts; no mention of } \\
\text { mole }\end{array}$ & N/A & $\operatorname{arr}(1-22) \times 3,(\mathrm{X}) \mathrm{x} 2,(\mathrm{Y}) \mathrm{x} 1$ \\
37 & $\begin{array}{l}\text { Fetal demise; follow up and autopsy } \\
\text { declined }\end{array}$ & N/A & $\operatorname{arr}(1-22, \mathrm{X}) \times 3$ \\
38 & $\begin{array}{l}\text { Termination; placenta grossly normal } \\
\text { N } 3 / \mathrm{A}\end{array}$ & $\operatorname{arr}(1-22, \mathrm{X}) \times 3$ \\
\hline
\end{tabular}

Among the 6 amniotic fluid specimens positive for triploidy, only 1/6 (17\%) was reported to be PHM-positive and hCG-elevated. N/A: no information available. 